\title{
Proposta de uma heurística construtiva baseada na TOC para definição de mix de produção
}

\author{
Vinicius Amorim Sobreiro ${ }^{\text {a* }}$, Marcelo Seido Nagano ${ }^{\mathrm{b}}$ \\ a*sobreiro@unb.br, FACE/UnB, Brasil \\ bdrnagano@usp.br, EESC/USP, Brasil
}

\begin{abstract}
Resumo
A definição do mix de produção proporciona a alocação dos recursos produtivos no processo de manufatura, visando a otimização da sua utilização e do desempenho do sistema produtivo. Entretanto, apesar de sua importância, a definição do mix de produção é um problema de difícil solução. Assim, com o auxílio da Teoria das restrições - TOC, algumas heurísticas construtivas têm sido apresentadas para fazer frente a esse problema. Nesse sentido, o objetivo neste trabalho é propor uma nova heurística que proporcione melhores soluções quando comparada com a heurística TOC-AK, de Aryanezhad e Komijan (2004, p. 4221-4233). Como resultado, observou-se que a heurística proposta obteve uma aproximação mais satisfatória quando comparada à aplicação da TOC-AK e à solução ótima identificada pela técnica de Programação Linear Inteira - PLl, o que, em conclusão, evidencia a importância da mesma na definição do mix de produção.
\end{abstract}

Palavras-chave

Heurística. TOC. Mix de produção.

\section{Introdução}

Em um problema de definição do mix de produção deve-se determinar em que quantidade cada um dos produtos escolhidos deve ser fabricado, com o objetivo de maximizar o ganho total, considerando que esses produtos podem ou não utilizar diversos recursos produtivos e que eles não são suficientes para a fabricação de todos os produtos. De acordo com Lea (2007, p. 1189-1190), o problema de definição do mix de produção pode ser representado da seguinte maneira:

$$
\begin{array}{ccc}
\text { Maximizar } & Z=\sum_{j=1}^{n}\left(P_{j}-c_{j}\right) x_{j} & \\
\text { Sujeito } & \sum_{j=1}^{n} a_{i j} x_{j} \leq b_{i} & i=1,2, \ldots, m \\
& x_{j} \leq d_{j} & j=1,2, \ldots, n
\end{array}
$$

Onde:

- $P_{j}$ representa o preço de venda do produto $j$;

- $c_{j}$ representa o custo do produto $j$;

- $\mathrm{a}_{i j}$ representa a quantidade de recurso $i$ requerida para produzir o produto $j$;
- $b_{i}$ representa a quantidade máxima disponível do recurso produtivo $i$;

- $x_{j}$ representa a quantidade do produto $j$ a ser produzido;

- $d_{j}$ representa a demanda de mercado prevista para o produto $j$;

- $m$ representa a quantidade de produtos; e

- $n$ representa a quantidade de recursos produtivos.

Em termos amplos, essa falta de recursos para a produção de todas as unidades demandadas pelo mercado acontece principalmente quando as organizações passam por períodos de ajuste estratégico ou de investimentos, pois mesmos as organizações precisam aumentar ou diminuir o nível de produção. Consequentemente, visto que a definição do mix de produção está diretamente relacionada com os ganhos a serem aferidos pela organização e que existem diversas opções de combinações de produtos que podem atender às restrições dos recursos produtivos, a melhor solução para esse tipo de situação é a otimização da sua utilização. Entretanto, como muito 
bem salienta Linhares (2009, p. 122), apesar de a definição das quantidades a serem produzidas, ou melhor, do mix de produção parecer um problema de fácil solução, com a inserção de novos produtos no processo de fabricação, o número de possibilidades de mix de produção aumenta exponencialmente o que, por sua vez, dificulta na obtenção do mix de produção ótimo em curto espaço de tempo.

Para fazer frente a essa situação, algumas heurísticas construtivas baseadas na Teoria das Restrições vêm sendo empregadas, visto que as mesmas proporcionam a definição do mix de produção com bons ganhos totais de maneira rápida. Entre essas heurísticas se destacam a TOC-h, de Fredendall e Lea, e sua sucessora, a TOC-AK, de Aryanezhad e Komijan. Entretanto, apesar de ambas heurísticas apresentarem bons resultados na definição do mix de produção em problemas com recursos produtivos insuficientes, não apresentam o mesmo desempenho em problemas que apresentam recursos produtivos insuficientes para atender a demanda de mercado dos produtos. Com base nesse contexto, o objetivo neste artigo é apresentar uma nova heurística construtiva que proporcione melhores soluções quando comparada à TOC-AK. Essa nova heurística difere da TOC-AK principalmente por levar em consideração apontamentos da TOC e do problema da mochila. Para realizar tal comparação, foram realizadas simulações computacionais visando identificar o mix de produção que possibilitasse o maior ganho possível, considerando um bom tempo de processamento e as características do ambiente produtivo.

Com base nesse contexto, além dessa introdução, este artigo está estruturado como se segue: na próxima seção, de maneira resumida, são definidos os conceitos básicos referentes à TOC e ao problema da mochila por meio de uma breve revisão de literatura. $\mathrm{Na}$ terceira seção, a heurística TOC-AK e a heurística proposta são demonstradas. Na seção seguinte, a experimentação computacional e os resultados obtidos são apresentados. Finalmente, na última seção, as principais conclusões e sugestões a trabalhos futuros são expostas.

\section{Revisão da literatura}

\subsection{Teoria das Restrições - TOC}

A Teoria das Restrições - TOC foi proposta e desenvolvida pelo Dr. Eliyahu M. Goldratt por volta de 1980 (LEA; FREDENDALL, 2002, p. 281). De maneira mais precisa, segundo Meleton (1987) apud Verma (1997, p. 191), a TOC surgiu em lsrael quando o Dr. Eliyahu M. Goldratt aplicou uma técnica de predição de átomos de cristal aquecido em problemas de programação de tarefas com grande número de variáveis. Em termos amplos, a TOC é compreendida como um conjunto de princípios teóricos que fundamentam e sintetizam a miríade de conhecimentos particulares de gestão e controle da produção que, por sua vez, reconhece o papel dos fatores limitantes nas operações de manufatura e foca-se neles, visando o aumento ou a melhoria de sua utilização. Ainda nesse contexto, utilizando-se das palavras de Watson, Blackstone e Gardiner (2007, p. 400), a TOC pode ser compreendida como:

[...] uma abordagem pragmática (prática) e holística (prioriza o entendimento integral dos fenômenos) de melhoria contínua, cobrindo com base em uma comum fundamentação teórica os fatores que limitam o aumento da performance em relação a uma meta. Assim, existe uma necessidade elevada de compreensão de técnicas específicas e das variáveis do sistema para assegurar o sucesso de sua implementação e ampla aceitação.

As características apontadas por esses pesquisadores são possíveis porque a TOC é capaz de auxiliar em diversas atividades fundamentais nas organizações as quais, por sua vez, contribuem para o desempenho global da mesma (FINCH; LUEBBE, 2000, p. 1466). De acordo com Corbett (2005, p. 35-36), a característica mais importante da TOC é assumir que em qualquer sistema existe no mínimo uma restrição, ou seja, alguma coisa que limita um melhor desempenho ou o alcance de altos padrões de desempenho. A afirmação de que todo sistema tem uma restrição se justifica ou é explicada pelo fato de que se não houvesse algo que limitasse o desempenho do sistema esse seria infinito. Nesse sentido, a gestão ou utilização de maneira eficiente das restrições do sistema permite melhorar o seu desempenho, pois somente os recursos gargalos, isto é, aqueles recursos que apresentam capacidade inferior à demanda, devem ser utilizados em sua total capacidade. Assim, é válido frisar que esse processo de gestão das restrições também facilita a compreensão e a possibilidade de otimização do sistema para os gestores organizacionais (MADAY, 1994, p. 84). De maneira mais pormenorizada, como muito bem destacam Antunes Junior e Rodrigues (1993, p. 80-81), esse processo de gestão das restrições é realizado pela utilização de cinco passos de melhoria comumente denominados processos de melhoria contínua, a saber:

- Identificar as restrições do sistema;

- Decidir como explorar as restrições;

- Subordinar todos os elementos da produção à restrição identificada no passo anterior;

- Aumentar a capacidade das restrições do sistema; e

- Se nos passos anteriores as restrições forem superadas, voltar ao primeiro passo sem deixar que a inércia se torne uma restrição. 
Considerando esses cincos passos, a TOC auxilia os gestores na condução dos processos que utilizam os recursos gargalos, ou seja, visa otimizar o emprego dos recursos gargalos nas atividades produtivas. De acordo com Goldratt e Cox (2006, p. 353) e Watson, Blackstone e Gardiner (2007, p. 338), essa abordagem de gestão sobre as restrições fez com que a TOC fosse implementada em grandes companhias como, por exemplo, 3M, Amazon, AVCO, Bendix, Boeing, Delta Airlines, Ford Motor Company, General Electric, General Motor, Kodak, Philips, RCA, Westinghouse e, também, em organizações sem fins lucrativos, como British National Health Service, Israel Air Force, NASA, Pretoria Academic Hospital e United States Department of Defense.

\subsection{Problema da mochila}

Em sua versão mais simplista, de acordo com Martello e Toth (1990, p. 1), Pisinger e Toth (1998, p. 302) e Kellerer, Pferschy e Pisinger (2004, p. 2-3), o problema da mochila pode ser matematicamente expresso pela definição, representando os objetos 1 até $n$, de um vetor de variáveis binárias $x_{j}(j=1, \ldots, n)$, com as características apresentadas na expressão 1:

$$
x_{j}=\left\{\begin{array}{cc}
1, & \text { seoobjetoé selecionado } \\
0, & \text { caso contrário }
\end{array}\right.
$$

Considerando que $p_{j}$ representa uma característica como, por exemplo, valor ou conforto do objeto $j, w_{j}$ corresponde ao tamanho desse objeto e $c$ ao tamanho da mochila, o problema será selecionar, entre todos os vetores binários, os $x_{j}$ que satisfaçam a restrição demonstrada na expressão 2 :

$$
\sum_{j=1}^{n} w_{j} x_{j} \leq c
$$

Além disso, a escolha dos $x_{j}$ deve visar a maximização da função objetivo mostrada na expressão 3:

$$
\sum_{j=1}^{n} p_{j} x_{j}
$$

Kellerer, Pferschy e Pisinger (2004, p. 15) sugerem um algoritmo do tipo Greedy baseado na seleção de produtos com maior eficiência $\left(e_{j}\right)$ para encontrar boas soluções. A $e_{j}$ é calculada com base na expressão 4:

$$
e_{j}=\frac{p_{j}}{w_{j}}
$$

Assim, ainda com base em Kellerer, Pferschy e Pisinger (2004, p. 16), a idéia do algoritmo é iniciar a escolha dos itens com a mochila vazia e ir acrescentando os itens que apresentam maior eficiência a ela, até que se alcance o limite da capacidade da mochila. O pseudocódigo desse algoritmo é apresentado na Figura 1.

No tocante ao mix de produção, esse algoritmo Greedy - considerando que a capacidade da mochila é análoga à capacidade disponível no recurso gargalo; que $p_{j}$ representa o ganho individual do produto $j$; e $w_{j}$ corresponda ao consumo de capacidade do recurso gargalo pelo produto $j$, apenas indicaria se um produto deve ou não ser produzido, mas seria incapaz de fornecer as quantidades a serem produzidas. Cabe destacar, no que diz respeito à TOC, que ela poderia ser utilizada para identificar o recurso gargalo e, tão logo, os valores de $e_{j}$, ou seja, da eficiência de cada produto. Assim, há necessidade de se adaptar o algoritmo Greedy para que ele seja capaz de indicar quais produtos deverão ser selecionados e em que quantidade cada um deles deve ser produzido. Essa versão adaptada do algoritmo Greedy, denominada $B$-Greedy, é apresentada na Figura 2.

0 algoritmo $B$-Greedy pode ser facilmente aplicado na definição do mix de produção desde que a TOC forneça a informação de qual recurso produtivo é o gargalo, aquele que deve orientar a definição de prioridade de processamento dos produtos, ou seja, qual recurso produtivo representará a mochila e poderá ser considerado para calcular as taxas de eficiência. Com base nesse contexto, visando relacionar os elementos da TOC com os do problema da mochila no enfoque da definição de mix de produção, o relacionamento adotado neste trabalho é apresentado na Tabela 1.

$$
\begin{array}{lll}
\text { 1. } & \bar{w}:=0 & \bar{w} \text { é o peso total da mochila com to } \\
\text { 2. } & Z^{G}:=0 & Z^{G} \text { é o ganho com a solução atual. } \\
\text { 3. } & \text { For } j:=1 \text { to } n \text { do } & \\
\text { 4. } & \text { If } \bar{w}+w_{j} \leq c \text { then } & \\
\text { 5. } & x_{j}:=1 & \\
\text { 6. } & \bar{w}:=\bar{w}+w_{j} & \\
\text { 7. } & Z^{G}:=Z^{G}+p_{j} & \\
\text { 8. } & \text { Else } x_{j}:=0 &
\end{array}
$$

$\bar{w}$ é o peso total da mochila com todos os itens.

Figura 1. Algoritmo Greedy. Fonte: Kellerer, Pferschy e Pisinger (2004, p. 16). 


$$
\begin{array}{lll}
\text { 1. } & \bar{w}:=0 & \bar{w} \text { é o peso total da mochila com todos os itens. } \\
\text { 2. } & Z^{G}:=0 & Z^{G} \text { é o ganho com a solução atual. } \\
\text { 3. } & \text { For } j:=1 \text { to } n \text { do } & \\
\text { 4. } & \text { If } \bar{w}+w_{j} \leq \text { then } & \\
\text { 5. } & x_{j}:=\min \left\{b_{j} ;\left[(c-\bar{w}) / w_{j}\right]\right\} \text { Colocando o item } j \text { na mochila. } \\
\text { 6. } & \bar{w}:=\bar{w}+w_{j} x_{j} & \\
\text { 7. } & Z^{G}:=Z^{G}+p_{j} x_{j} & \\
\text { 8. } & \text { Else } x_{j}:=0
\end{array}
$$

Figura 2. Algoritmo B-Greedy. Fonte: adaptado de Kellerer, Pferschy e Pisinger (2004, p. 187).

Tabela 1. Relacionando os elementos do problema da mochila com os elementos da TOC.

\begin{tabular}{cl}
\hline Problema da mochila & \multicolumn{1}{c}{ Mix de produção } \\
\hline$j$ & Produto \\
$p_{j}$ & Ganho \\
$w_{j}$ & Consumo de recurso gargalo \\
$b_{j}$ & Demanda \\
$e_{j}$ & Eficiência \\
$c$ & Capacidade disponível no gargalo \\
\hline
\end{tabular}

\section{Método heurístico}

\subsection{Notação}

Para os métodos heurísticos apresentados nesta seção, a seguinte notação é utilizada:

- Indices

- $i=1,2, \ldots, n$ Produtos;

- $j=1,2, \ldots, m$ Recursos produtivos; e

- $q=1,2, \ldots, m$ Recursos gargalo.

- Variáveis de decisão

- $B N_{q}$ Recurso gargalo $q$, ou seja, todos os recursos que apresentaram $d_{j}<0$;

- $C P_{j}$ Capacidade produtiva do recurso $j$;

- $C M_{i}$ Margem de contribuição do produto $i$;

- $C R$ Vetor contendo os recursos gargalos;

- $d_{j}$ Diferença entre a capacidade disponível e a necessária no recurso produtivo $j$;

$D_{i} \quad$ Demanda de mercado do produto $i$;

- $H$ Conjunto de produtos que não tiveram sua demanda atendida;

- $i$ Posição do produto que na sequência de produção terá sua quantidade aumentada;

- $k$ Posição do produto que na sequência de produção terá sua quantidade reduzida;

- $N$ Quantidade do produto $X$ que precisa ser reduzida para aumentar uma unidade de $Y$;

- $P$ Produto;

- $q$ Número de recursos gargalos;
- $R_{i}$ Relação entre a margem de contribuição e cada unidade de tempo de processamento consumida no recurso gargalo;

- $R_{i, B N_{k}}$ Relação entre a margem de contribuição e cada unidade de tempo de processamento consumida no recurso gargalo $B N_{k}$;

- $R A_{i}$ Soma do $R_{i}$ em todos os gargalos para o produto $i$;

- $t_{i j}$ Tempo de processamento ou consumo de recurso do produto $i$ no recurso produtivo $j$;

- $t_{\text {left }, q}$ Sobra de recurso disponível no recurso gargalo $q$;

- $x_{i} \quad$ Quantidade a ser produzida do produto $i$;

- $X$ Conjunto de produtos candidatos a sofrer redução na quantidade; e

- Z Parâmetro utilizado para indicar qual são a melhor alternativa de redução e de aumento entre as diversas opções de produtos existentes.

\subsection{Heurística TOC-AK}

A heurística TOC-AK, proposta por Aryanezhad e Komijan (2004, p. 4227), sucessora da heurística TOC-h proposta por Fredendall e Lea (1997, p. 1537), procura identificar o mix de produção observando todas as possíveis sequências de produção para cada gargalo. Para cumprir tal propósito, a heurística TOC-AK identifica os produtos que apresentam classificações diferentes em meios a todas as sequências de produção por gargalo e realiza uma análise verificando quais produtos e em quais quantidades eles devem ser produzidos. 0 pseudocódigo da heurística TOC-AK é apresentado a seguir:

Passo 1 - Identifique a(s) restrição(s) do sistema

Calcule a diferença $\left(d_{j}\right)$ entre a capacidade dos recursos e a demanda deles:

$$
d_{j}=C P_{j}-\sum_{i=1}^{n} t_{i j} D_{i} i=1,2, \ldots, n \text { e } j=1,2, \ldots, m
$$

Determine $C R=\left\{B N_{1}, B N_{2} \ldots, B N_{q}\right\}$, considerando que $d_{q} \leq 0$ e $d_{1} \leq d_{2} \leq \ldots \leq d_{q^{\circ}}$.

Passo 2 - Determine a prioridade da produção considerando cada gargalo 
Para cada gargalo, calcule $R_{i, B N_{k}}$ da seguinte maneira:

$$
R_{i, B N_{k}}=\frac{C M_{i}}{t_{i, B N_{k}}} i=1,2, \ldots, n \text { e } k=1,2, \ldots, q
$$

Assim, $R_{i, B N_{k}}$ representará a prioridade do produto $i$ na perspectiva do gargalo $k$. Repita os seguintes procedimentos para cada gargalo para determinar a prioridade de produto nele:

- Classifique os produtos que são processados no gargalo $k$ de maneira decrescente com base em $R_{i, B N_{k}}$. Classifique os produtos que apresentarem o mesmo $R_{i, B N_{k}}$ de maneira decrescente com base em $C M_{i}$;

- Classifique os produtos que não são processados no gargalo $k$ de maneira decrescente considerando a $C M_{i}$ Os produtos que apresentarem o mesmo $C M_{i}$ devem ser classificados de maneira arbitrária; e

- Classifique os produtos "livres" no final da lista de maneira decrescente com base em $C M_{i}$

Dessa forma, cada gargalo apresenta uma sequência de produção a ser seguida.

Passo 3 - Desenvolva um plano mestre de produção viável (MPS)

Programe os produtos de acordo com a sequência de produção determinada para $B N_{1}$ e o limite de capacidade dos outros gargalos. 0 processo de considerar a capacidade limite dos outros gargalos é inevitável para se alcançar uma programação de produção viável.

Passo 4 - Determine alternativas viáveis para aumentar o ganho

Examine se a redução da produção de um produto e o aumento de produção de outro produto poderá aumentar o ganho. Assuma que $X$ e $Y$ são os produtos candidatos para o processo de diminuição e aumento. A alternativa de se diminuir $X$ e aumentar $Y$ deve ser considerada válida caso as seguintes condições sejam encontradas:

- Se no plano mestre de produção viável (MSP), $X$ tiver prioridade sobre $Y$; e

- Se o produto $Y$ apresentar três condições: (a) sua demanda não foi atendida, (b) apresentar prioridade sobre $X$ em pelo menos uma das sequências, e (c) na mesma sequência em que tiver prioridade sobre $X$, tiver prioridade sobre todos os produtos cuja demanda não foi atendida.

Passo 5 - Selecionar a melhor alternativa

Se não foram desenvolvidas alternativas no passo 4 , a solução obtida no passo 3 deve ser a ótima. Se uma alternativa foi desenvolvida, vá para o passo 6. Por outro lado, se houver várias alternativas, identifique a melhor alternativa para alcançar a solução ótima.
Para esse propósito, repita os seguintes procedimentos, para cada alternativa:

- Considerando o tempo que sobrou nos gargalos, determine quantas unidades $(N)$ de $X$ deveriam ser reduzidas para aumentar uma unidade de $Y$. Então, calcule $Z$ da seguinte maneira:

$$
Z=\sum_{k=1}^{q \leq m}\left(-N R_{X, B N_{k}}+R_{Y, B N_{k}}\right)
$$

Escolher a alternativa com o maior valor de $Z$ e passar para o passo 6 .

Passo 6 - Desenvolva um conjunto com os produtos que não tiveram a demanda atendida

Classifique todos os produtos (exceto $Y$ ) que não tiveram sua demanda atendida de maneira decrescente com base em $C M_{i}$ e de o nome a esse conjunto de $H$. Os produtos que apresentarem o mesmo $C M_{i}$ devem ser classificados de maneira arbitrária.

Passo 7 - Determine o máximo de unidades de $X$ que podem ser reduzidas

Determine quantas unidades $(N)$ de $X$ poderão ser reduzidas sequencialmente para aumentar uma unidade de $Y$ com base nos seguintes procedimentos:

- Assuma que $Y$ tem prioridade sobre $X$ na sequência desenvolvida considerando o gargalo $k$;

Calcule $f=R_{Y, B N_{K}} / R_{X, B N_{K}}$;

- Determine quantas unidades de $X$ não serão processadas se o gargalo $k$ não processar $X$ por $f$ minutos $\left(f / t_{\chi, B N_{k}}\right)$;

- Determine quantas unidades de $Y$ podem ser processadas por $B N_{k}$ em um minuto $\left(1 / t_{Y, B N_{k}}\right)$; e

- Calcule quantas unidades $\left(N_{k}\right)$ de $X$ poderão ser reduzidas sequencialmente para aumentar uma unidade de $Y$, com base na seguinte expressão $N_{k}=\left(f / t_{\chi, B N_{k}}\right) /\left(1 / t_{Y, B N_{k}}\right)$. Se $N_{k}$ não for inteiro, arredonde para baixo.

Repita os procedimentos acima em todas as sequências em que $Y$ é prioritário a $X$. Faça $N$ como o máximo valor obtido de $N_{k}$. Observe que, no mínimo, a soma de $N$ será um, desde que pelo menos uma unidade de $X$ deva ser reduzida para aumentar $Y$. Então, caso $N$ se torna zero, conforme os cálculos acima, o mesmo deve ser ajustado para um.

Passo 8 - Processo de redução e aumento

Reduza uma unidade de $X$. Examine se o tempo que restou no gargalo possibilita o aumento de $Y$. Se isso for possivel, aumente $Y$ até que algumas dessas situações ocorram:

- A demanda de $Y$ é atendida: nesse caso, ajuste o primeiro membro de $H$ como $Y$ e volte ao passo 6;

- Não é possível aumentar $Y$ por falta de capacidade: deve-se examinar a possibilidade de aumentar o primeiro membro de $H$. Se sua demanda já foi 
atendida ou não existir possibilidade de aumentar as quantidades, proceda para o próximo membro do grupo e repita esse procedimento para todos os membros de $H$.

Se isso não for possível, repita o procedimento 8 . Pare se não for possivel aumentar $Y$ depois de reduzir $N$ unidades de $X$.

\subsection{Heurística proposta}

A heurística proposta visa a criação de uma solução inicial considerando o gargalo dominante e, tão logo, a sua melhoria. Esse processo de criação da solução inicial é realizado com o auxílio do algoritmo $B$-Greedy para problemas em que o gargalo dominante é identificado e de uma versão modificada do mesmo, denominada de $B$-Greedy- $M$, para problemas em que a identificação do gargalo dominante é difícil ou impossível. A utilização do algoritmo $B$-Greedy ou do $B$-Greedy- $M$ possibilita que a heurística proposta encontre uma solução inicial de maneira muito rápida o que, por sua vez, possibilita utilizar mais tempo na tentativa de melhorar a solução inicial.

Esse processo de melhoria é realizado mediante uma busca na vizinhança que considera diferentes produtos em diferentes posições, na sequência de produção. Além disso, também visando a melhoria da solução inicial, é proposta a utilização de uma taxa de ganho que leve em conta o ganho por tempo de processamento do produto $i$ em todos os gargalos existentes de maneira conjunta e não somente no gargalo dominante, pois nem sempre é possível identificar o gargalo dominante. Consequentemente, a heurística adota como solução final ou mix de produção a melhor solução encontrada após a realização dessa busca na vizinhança. Com base nesse contexto se faz necessário apresentar primeiramente o algoritmo $B$-Greedy- $M$ para então expor o pseudocódigo da heurística proposta, visto que a conceituação e utilização do $B$-Greedy já foi demonstrada na Seção 2.2.

\subsubsection{O algoritmo B-Greedy-M}

Em alguns problemas de definição do mix de produção é difícil ou impossível identificar o gargalo dominante em meio aos existentes. Essa situação não possibilita a aplicação do $B$-Greedy, visto que ele se baseia nas taxas de eficiência, ou seja, $e_{j}$ que, por sua vez, é obtida considerando o gargalo dominante. Assim se faz necessário ajustar o $B$-Greedy para que seja identificado um mix de produção, dado a existência de vários recursos gargalos e/ou a dificuldade de identificação do recurso gargalo dominante. Com base nesse contexto, o algoritmo
$B$-Greedy- $M$ considera uma sequência de produção comum em todos os gargalos e busca identificar as quantidades máximas que cada gargalo possibilita produzir de um determinado produto, obedecendo à sequência de produção. Posteriormente, o mesmo assume como quantidade a ser produzida de um determinado produto a menor quantidade encontrada entre todos os gargalos. 0 pseudocódigo do algoritmo $B$-Greedy-M é apresentado na Figura 3.

\subsubsection{O pseudocódigo da heurística proposta}

Considerando o algoritmo B-Greedy e o $B$-Greedy- $M$, o pseudocódigo da heurística proposta é apresentado a seguir:

Passo 1 - Identifique a(s) restrições(s) do sistema

- Calcule a diferença $\left(d_{j}\right)$ entre a capacidade dos recursos e a demanda prevista eles:

$$
\begin{aligned}
d_{j}=C P_{j}-\sum_{i=1}^{n} t_{i j} D_{i} \quad j & =1,2, \ldots, n \\
j & =1,2, \ldots, m
\end{aligned}
$$

- Considerando que todos os recursos que apresentarem $d_{j}<0$ serão considerados recursos gargalos $\left(B N_{q}\right)$, determine $C R=\left\{B N_{1}, B N_{2} \ldots, B N_{q}\right\}$, observando que $d_{q} \leq 0$ e $d_{1} \leq d_{2} \leq \ldots \leq d_{q}$.

Passo 2 - Decida como explorar a(s) restrições(s) do sistema

- a) Assuma que $B N_{1}$ é o gargalo dominante. Calcule para cada produto a taxa de $R_{i}$, ou seja, a margem de contribuição por cada unidade de tempo de processamento consumida no gargalo, pela expressão

$$
\begin{aligned}
& \text { 1. } \overline{w_{q}}:=0 \\
& \text { 2. } Z:=0 \\
& \text { 3. For } i:=1 \text { to } n \text { do } \\
& \text { 4. For } q:=1 \text { to } m \text { do } \\
& \text { If } \overline{w_{q}}+w_{i q} \leq c_{q} \text { then } \\
& \text { 6. If } w_{i q}=0 \text { then } \\
& 8 . \\
& 8 . \\
& 11 . \\
& 12 . \\
& 13 . \\
& 14 . \\
& 15 . \\
& 6 . \\
& 17 . \\
& 18 . \\
& 20 . \\
& 21 \\
& 21 . \\
& 22 . \\
& 24 . \\
& 25 . \\
& 26 . \\
& \text { Next } 9 \\
& \text { Else } \\
& \text { End if } \\
& x_{i q}:=\operatorname{int}\left\{\min \left\{D_{i} ;\left[\left(c_{q}-\overline{w_{q}}\right) / w_{i q}\right]\right\}\right\}
\end{aligned}
$$

Figura 3. Algoritmo B-Greedy-M. 
$R_{i}=C M_{i} / t_{i, B N_{1}}$. Classifique os produtos de maneira decrescente com base no valor $R_{i}$ obtido.

- b) Verifique se o $B N_{1}$ é o gargalo dominante:

1. Aplique o algoritmo B-Greedy ao problema considerando o $R_{i}$ obtido em passo 2 a e a sequência de produção atual.

11. Considerando o mix de produção obtido no passo $2 \mathrm{~b} .1$, examine se existe alguma $d_{j}$ menor do que zero, caso exista assuma o próximo $B N$ em $C R$ como $B N_{1}$, classifique os produtos com base nesse novo recurso gargalo e volte para o passo 2a. Se o $B N_{1}$ for igual a $B N_{q}$ e ainda existir $d_{j}$ menor que zero vá para o passo 2 b.lll. Por outro lado, se não existir nenhum $d_{j}$ menor que zero vá para o passo $2 \mathrm{c}$.

111. Aplique o algoritmo $B$-Greedy- $M$ considerando a sequência de produção dada por $B N_{q}$ para obter o mix de produção que não apresente $d_{j}$ menor que zero.

- c) Assuma o ganho total obtido como $S_{1}$.

- d) Verifique a possibilidade de melhorar a solução obtida:

1. Identifique na sequência de produção que proporcionou $S_{1}$ o último produto com o maior $R_{i}$ que apresenta produção igual a demanda, isto é, $x_{i}=D_{i}$. Reduza a demanda desse produto, uma unidade por vez, e aplique o $B$-Greedy- $M$ considerando a classificação dos produtos dada por $R_{i}$, visando identificar um novo mix de produção. Realize esse procedimento até que a demanda de $i$ seja igual a $D_{i}-D_{i} \times 0,2$ ou não seja possível aumentar as quantidades a serem produzidas dos demais produto. Caso existam dois produtos com o mesmo valor de $R_{i}$, sequencie o de maior $C M_{i}$ primeiro. Assuma como $S_{2}$ o maior ganho tortal obtido na realização desse procedimento.

11. Calcule o $R A_{i}$ para todos os produtos da seguinte forma:

$$
R A_{i}=\sum_{g=1}^{q} C M_{i} / t_{i, B N_{g}}
$$

Classifique os produtos de maneira decrescente com base no $R A_{i}$ e se existir produtos com o mesmo valor de $R A_{i}$, considere primeiramente o que apresentar o maior $C M_{i}$. Com base nessa nova classificação aplique o algoritmo $B$-Greedy- $M$, visando identificar um novo mix de produção inicial. Caso essa nova sequência de produção seja idêntica à sequência anterior, proceda para o passo 2 d.11l. Caso contrário, iniciando a procura do produto com maior $R A_{i}$ para o menor, identifique o último produto que apresentou $x_{i}=D_{i}$. Reduza a demanda desse produto, uma unidade por vez, e sequencie a produção com base nessa nova demanda até que ela seja igual $D_{i}-D_{i} \times 0,1$ ou não seja possível aumentar as quantidades a serem produzidas dos demais produtos. Comparando os resultados obtidos entre o novo mix de produção e os mixes de produção proporcionados pela redução da demanda do produto $x_{i}$, assuma o maior ganho total obtido como $S_{3}$.

111. Considerando o mix de produção obtido na solução $S_{1}$, identifique o produto que apresentar o maior $R_{i}$. Reduza a demanda desse produto, uma unidade por vez, e sequencie a produção com base nessa nova demanda, utilizando o $B$-Greedy- $M$. Realize esse procedimento até que a demanda desse produto seja igual à $D_{i}-D_{i} \times 0,1$ ou não seja possivel aumentar as quantidades a serem produzidas dos demais produtos. Assuma o maior ganho total encontrado como $S_{4}$.

IV. Se o gargalo dominante for $B N_{1}$, faça $S_{5}$ igual a zero e proceda para o passo 2.e. Caso contrário, sequencie os produtos com base no $R_{i}$ do primeiro gargalo do conjunto $C R$, ou seja, na maior diferença de capacidade existente. Caso existam produtos com o mesmo valor de $R_{i}$, sequencie o que apresentar maior $C M$ primeiro. Aplique o algoritmo $B$-Greedy- $M$ visando identificar um mix de produção. Com base nele, identifique o último produto que apresentar $x_{i}=D_{i}$, diminua uma unidade por vez da demanda desse produto e sequencie novamente todos os produtos. Realize esse procedimento até que a demanda desse produto seja igual a $D_{i}-D_{i} \times 0,1$ ou não seja possível aumentar as quantidades a serem produzidas dos demais produtos. Comparando os resultados obtidos entre o mix de produção obtido com base em $B N_{1} \mathrm{e}$ os mixes de produção proporcionados pela redução da demanda do produto $x_{i}$, assuma o maior ganho total obtido como $S_{5}$.

- e) Assuma como solução final da heurística o mix de produção que apresentar o maior ganho do seguinte conjunto $\left\{S_{1}, S_{2}, S_{3}, S_{4}, S_{5}\right\}$.

No tocante às duas heurísticas é importante destacar, como muito bem salientado por Hsu e Chung (1998, p. 37), que as mesmas são baseadas na utilização dos passos 1 e 11 do processo de melhoria da TOC, pois os demais passos são ações gerenciais baseadas na solução desenvolvida na realização dos dois primeiros passos. Assim considerando o escopo deste trabalho, para fins de definição de mix de produção, não se faz uso dos demais passos.

\section{Experimento computacional}

Com o objetivo de testar as heurísticas apresentadas na seção 3, uma extensa experimentação computacional foi realizada visando identificar a melhor heurística. Todas as heurísticas foram testadas na resolução de problemas encontrados na literatura especializada e 
gerados aleatoriamente. Os problemas foram divididos em dois grupos, a saber:

- Pequeno porte: constituído por 100 problemas contendo $i$ de 2 até $8, j$ de 4 até 8 , e $q$ de 1 até 4 ; e

- Grande porte: constituído por 50 problemas contendo $i$ igual a 100, $i$ de 60 até 100, e $q$ de 6 até 60 .

Além disso, todas as heurísticas foram desenvolvidas em VBA e testadas em computador lntel $2.16 \mathrm{GHz}$ Dual Clock, com 4 GB de memória RAM. Visando a comparação entre as heurísticas, o tempo total de processamento e o desvio relativo médio - DRM foram utilizados como medidas de comparação. 0 DRM foi calculado conforme a expressão 5.

$$
D R M=\frac{\left(f(h)-f^{*}\right)}{f^{*}} \times 100
$$

onde

- $f(h)$ é o ganho obtido pela heurística $h$, ou seja, a TOC-AK ou a heurística proposta; e

- $f^{*}$ representa a melhor solução obtida entre ambas as heurísticas.

Considerando os problemas de pequeno porte, o resumo dos resultados obtidos pelas heurísticas para problemas com 1, 2, 3, e 4 gargalos é apresentado na Tabela 2.

Além disso, considerando os problemas de pequeno porte, as médias com um intervalo de confiança de 99\% são apresentadas para cada heurística na Figura 4.

Com base na Tabela 2 e na Figura 4, a heurística proposta apresentou um melhor desempenho do que a heurística TOC-AK nos problemas de pequeno porte, pois na maioria dos casos apresentou o resultado maior ou um resultado muito próximo do maior. Quanto ao tempo de processamento, visto o tamanho dos problemas, ambas heurísticas são rápidas, isso é, necessitam de menos de um segundo para resolver os problemas o que, por sua vez, não possibilita identificar diferenças significativas entre as mesmas. No tocante aos problemas de grande porte, o resumo dos resultados obtidos está apresentado na Tabela 3.

Conforme apresentado na Tabela 3, a heurística proposta também apresenta um desempenho melhor que a TOC-AK em problemas de grande porte, visto que apresentou em média um DRM de aproximadamente $0,78 \%$ em relação à melhor solução encontrada entre ambas heurísticas e utilizou em média 32 segundos para resolver os problemas agrupados pelo percentual de gargalos existentes. No tocante à TOC-AK, os resultados indicam que a qualidade da solução só foi melhorada devido à utilização de quantidades maiores de tempo de processamento. É importante destacar que esse apontamento se torna mais expressivo quando a quantidade de recursos gargalo aumenta no ambiente produtivo, ou seja, quando há muitos recursos gargalo no sistema produtivo a TOC-AK despende muito tempo criando e comparando sequências de produção por recursos gargalo. Em contrapartida, a heurística proposta utiliza pouco tempo na criação da solução inicial e mais tempo na melhoria dela. Essa característica proporciona à heurística proposta uma

Tabela 2. Comparação dos resultados obtidos para os problemas de pequeno porte em termos da média do DRM e do tempo total de processamento.

\begin{tabular}{|c|c|c|}
\hline \multirow{2}{*}{ Número de gargalos } & \multicolumn{2}{|c|}{ Heuristicas } \\
\hline & TOC-AK & Proposta \\
\hline 1 & $-0,1818^{\mathrm{A}}(00: 00: 00)^{\mathrm{B}}$ & 0,0000 (00:00:00) \\
\hline 2 & $-1,2995$ (00:00:00) & $-0,0081$ (00:00:00) \\
\hline 3 & $-2,5564$ (00:00:00) & $-0,5968(00: 00: 02)$ \\
\hline 4 & $-5,6760(00: 00: 01)$ & $-0,6278(00: 00: 00)$ \\
\hline Média & $-2,4284(00: 00: 00)$ & $-0,3082(00: 00: 01)$ \\
\hline
\end{tabular}

${ }^{\mathrm{A}}$ Média do desvio relativo médio; ${ }^{\mathrm{B}}$ Total de tempo necessário para processar todos os problemas com esse número de gargalos.

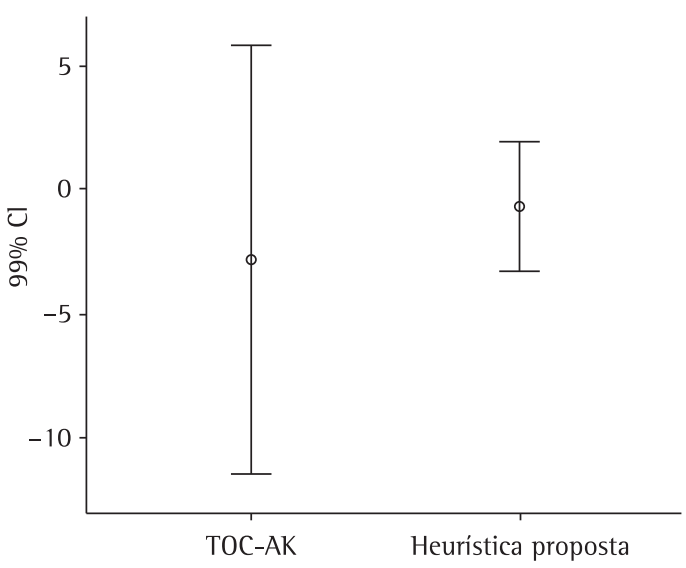

Figura 4. Média e intervalo de confiança (99\%) das heurísticas para os problemas de pequeno porte.

Tabela 3. Comparação dos resultados obtidos para os problemas de grande porte em termos do DRM e do tempo total de processamento.

\begin{tabular}{ccr}
\hline \multirow{2}{*}{$\begin{array}{c}\text { \% de recursos } \\
\text { gargalo }\end{array}$} & \multicolumn{2}{c}{ Heuristicas } \\
\cline { 2 - 3 } & \multicolumn{1}{c}{ TOC-AK } & \multicolumn{1}{c}{ Proposta } \\
\hline 10 & $-1,6644^{\mathrm{A}}(00: 00: 29)^{\mathrm{B}}$ & $-3,0654(00: 00: 06)$ \\
20 & $-0,0814(00: 00: 58)$ & $-0,1237(00: 00: 13)$ \\
30 & $-0,0903(00: 00: 44)$ & $-1,1008(00: 00: 16)$ \\
40 & $-13,9732(00: 01: 21)$ & $0,0000(00: 00: 24)$ \\
50 & $-4,7471(00: 02: 20)$ & $-2,7705(00: 00: 23)$ \\
60 & $-12,0273(00: 01: 49)$ & $-0,0108(00: 00: 29)$ \\
70 & $-11,6532(00: 03: 05)$ & $0,0000(00: 00: 36)$ \\
80 & $-13,2038(00: 03: 43)$ & $0,0000(00: 00: 51)$ \\
90 & $-15,0692(00: 04: 25)$ & $0,0000(00: 00: 59)$ \\
100 & $-13,4203(00: 03: 41)$ & $-0,6567(00: 01: 02)$ \\
Média & $-8,5930(00: 02: 15)$ & $-0,7728(00: 00: 32)$ \\
\hline
\end{tabular}

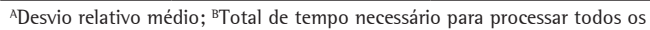
problemas com esse percentual de gargalos. 
menor variabilidade em relação à média do DRM do que a TOC-AK, conforme apresentado na Figura 5.

Considerando que a heurística proposta apresentou um melhor desempenho tanto para os problemas de pequeno quanto para os de grande porte, ela foi comparada com as soluções ótimas obtidas por meio da técnica de PLI. Para tanto foi definido um tempo máximo de 24 horas para realização desse processo

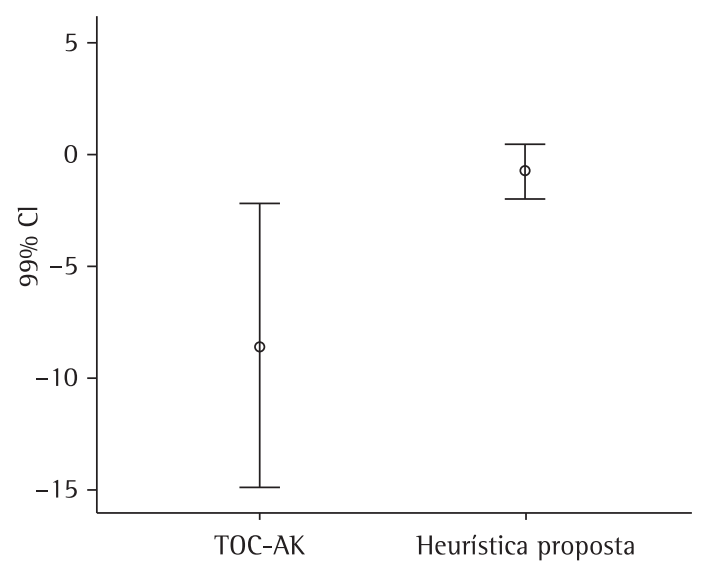

Figura 5. Média e intervalo de confiança (99\%) das heurísticas para os problemas de grande porte

Tabela 4. Comparação considerando o DRM entre a heurística proposta e a técnica de PLI.

\begin{tabular}{cccc}
\hline \multirow{2}{*}{$\begin{array}{c}\text { Número de } \\
\text { gargalos }\end{array}$} & DRM & \multicolumn{2}{c}{ Tempo de processamento total } \\
\cline { 2 - 4 } & $\begin{array}{c}\text { Heuristica } \\
\text { proposta }\end{array}$ & $\begin{array}{c}\text { Heuristica } \\
\text { roposta }\end{array}$ & PLl \\
\hline 1 & 0,0000 & $00: 00: 00$ & $00: 00: 00$ \\
2 & $-0,4688$ & $00: 00: 00$ & $00: 00: 13$ \\
3 & $-2,4955$ & $00: 00: 02$ & $00: 00: 05$ \\
4 & $-3,8021$ & $00: 00: 00$ & $00: 00: 04$ \\
Média & $-1,6916$ & $00: 00: 01$ & $00: 00: 06$ \\
\hline
\end{tabular}

Tabela 5. Comparação considerando o DRM entre a heurística proposta e a técnica de PLI.

\begin{tabular}{cccc}
\hline \multirow{2}{*}{ \% de gargalos } & DRM & \multicolumn{2}{c}{ Tempo de processamento total } \\
\cline { 2 - 4 } & $\begin{array}{c}\text { Heuristica } \\
\text { proposta }\end{array}$ & $\begin{array}{c}\text { Heurística } \\
\text { proposta }\end{array}$ & PL1 \\
\hline 10 & $-29,4855$ & $00: 00: 06$ & $00: 32: 23$ \\
20 & $-19,6491$ & $00: 00: 13$ & $00: 29: 38$ \\
30 & $-19,1612$ & $00: 00: 16$ & $00: 33: 54$ \\
40 & $-12,9456$ & $00: 00: 24$ & $00: 17: 08$ \\
50 & $-20,7603$ & $00: 00: 23$ & $00: 47: 29$ \\
60 & $-14,5410$ & $00: 00: 29$ & $02: 41: 36$ \\
70 & $-17,8203$ & $00: 00: 36$ & $01: 00: 04$ \\
80 & $-13,9182$ & $00: 00: 51$ & $03: 02: 37$ \\
90 & $-11,6786$ & $00: 00: 59$ & $24: 42: 32^{\AA}$ \\
100 & $-13,1521$ & $00: 01: 02$ & $01: 24: 41$ \\
Média & $-17,3112$ & $00: 00: 32$ & $03: 33: 12$ \\
\hline
\end{tabular}

${ }^{\wedge}$ Nesse conjunto de problemas houve um em que a técnica PLI não obteve a solução ótima depois de 24 horas. de procura da solução ótima em cada problema, visto que tal processo de busca pela solução ótima pode ser um processo moroso, ou seja, caso a técnica de PLl utilizasse 24 horas para encontrar a solução ótima e não a encontrasse, a melhor solução encontrada seria adotada. Com base nesse contexto, assumindo que os valores obtidos pela técnica de PLl são $f^{*}$, na Tabela 4 são apresentados os DRM da heurística proposta e o tempo total de processamento para os problemas de pequeno porte e, na Tabela 5, esses resultados são apresentados para os problemas de grande porte.

A heurística proposta apresentou na média um DRM de aproximadamente 17,32\% em relação aos resultados ótimos. Apesar de esse valor ser expressivo, houve uma diminuição significativa na média do tempo total de processamento utilizado para resolução desses problemas o que, por sua vez, evidência a eficiência e robustez da heurística proposta.

Ainda no tocante ao desempenho da heurística proposta, Linhares $(2009$, p. 128) aponta que um dos principais problemas ou falhas das heurísticas desenvolvidas para os problemas de definição de mix de produção, apesar de sua eficiência na solução de problemas mais complexos, é a dificuldade de obtenção das soluções ótimas para problemas com apenas um gargalo. Entretanto, conforme exposto na Tabela 4, a heurística proposta não apresenta essa dificuldade, pois conseguiu encontrar todas as soluções ótimas para esses problemas mais simples.

\section{Conclusões}

Neste artigo foi tratado o problema de definição de mix de produção com o objetivo de se maximizar o ganho, considerando a teoria das restrições, por meio de heurísticas construtivas. De maneira prática, tal problema pode ser compreendido como a definição das quantidades a serem produzidas de cada produto quando os recursos produtivos são insuficientes para atender a toda demanda. Entretanto, a busca de soluções próximas à ótima pelo uso de eficientes e simples heurísticas ainda necessita de pesquisas, visto que esse problema é do tipo NP-Completo. Dentro desse contexto, foi proposta uma nova heurística para esse problema com base na TOC e no problema da mochila que apresenta melhor desempenho, em termos de qualidade de solução, que a heurística TOC-AK, de Aryanezhad e Komijan (2004, p. 4221-4233). Assim, pode-se concluir que a heurística proposta é muito mais eficiente que as heurísticas existentes o que, em suma, destaca sua importância na definição de mix de produção. No tocante à indicação de pesquisas futuras, é proposta 
a realização de estudos que verifiquem a utilização da heurística proposta em situação prática.

\section{Referências}

ANTUNES JUNIOR, J. A. V.; RODRIGUES, L. H. A teoria das restrições como balizadora das ações visando a troca rápida de ferramentas. Produção, v. 3, n. 2, p. 73-85, 1993.

ARYANEZHAD, M. B.; KOMIJAN, A. R. An improved algorithm for optimizing product mix under the theory of constraints. International Journal of Production Research, v. 42, n. 20, p. 4221-4233, 2004. http://dx.doi.org/10.1080/0020754 0410001695961

CORBETT, T. Bússola Financeira: 0 processo decisório da Teoria das Restrições. São Paulo: Nobel, 2005. 208 p.

FINCH, B. J.; LUEBBE, R. L. Response to 'Theory of constraints and linear programming: a re-examination'. International Journal of Production Research, v. 38, n. 6, p. 1465-1466, 2000. http://dx.doi.org/10.1080/002075400188960

FREDENDALL, L. D.; LEA, B. R. Improving the product mix heuristic in the theory of constraints. International Journal of Production Research, v. 35, n. 6, p. 1535-1544, 1997. http://dx.doi.org/10.1080/002075497195100

GOLDRATT, E. M.; COX, J. A meta: um processo de melhoria contínua. 2. ed. São Paulo: Nobel, 2006. 365 p.

HSU, T.-C.; CHUNG, S.-H. The TOC-based algorithm for solving product mix problems. Production Planning Et Control, v. 9, n. 1, p. 36-46, 1998. http://dx.doi. org/10.1080/095372898234505

KELLERER, H.; PFERSCHY, U.; PISINGER, D. Knapsack Problems. Berlin: Springer, 2004. 546 p.
LEA, B.-R. Management accounting in ERP integrated MRP and TOC environments. Industrial Management \&t Data Systems, v. 107, n. 8, p. 1188-1211, 2007. http://dx.doi. org/10.1108/02635570710822813

LEA, B.-R.; FREDENDALL, L. D. The impact of management accounting, product structure, product mix algorithm, and planning horizon on manufacturing perfomance. International Journal Production Economics, v. 79, n. 3, p. 279-299, 2002. http://dx.doi.org/10.1016/S09255273(02)00253-0

LINHARES, A. Theory of constraints and the combinatorial complexity of the product-mix decision. International Journal Production Economics, v. 121, n. 1, p. 121-129, 2009. http://dx.doi.org/10.1016/j.jpe.2009.04.023

MADAY, J. C. Proper use of constraint management. Production and Inventory Management Journal, v. 35, n. 1, p. 84, 1994.

MARTELLO, S.; TOTH, P. Knapsack problems: algorithms and computer implementations. Guildford: John Wiley \& Sons, 1990. 306 p.

PISINGER, D.; TOTH, P. Knapsack Problems. In: DU, D. Z.; PARDALOS, P. M. Handbook of Combinatorial Optimization. Boston: Kluwer Academic Publisher, 1998. v. 1, p. 299-428. http://dx.doi.org/10.1007/978-1-4613-0303-9_5

VERMA, R. Management Science, Theory of Constraints/ Optimized Production Technology and Local Optimization. Omega, v. 25, n. 2, p. 189-200, 1997. http://dx.doi. org/10.1016/S0305-0483(96)00060-6

WATSON, K. J.; BLACKSTONE, J. H.; GARDINER, S. C. The evolution of a management philosophy: The theory of constraints. Journal of Operations Management, v. 25, n. 2, p. 387-402, 2007. http://dx.doi.org/10.1016/j. jom.2006.04.004

\title{
A proposal of a constructive heuristics under the TOC for definition of production mix
}

\begin{abstract}
The definition the product mix enables the allocation of productive resources in the manufacturing process, aiming to optimize the use of productive resources and productive system performance. However, the definition of the product mix is a problem of difficult solution. Thus, with the help of the Theory of Constraints - TOC, some constructive heuristics were presented to help solve those problems. Taking this into account, the objective of this paper was to propose a new heuristics to provide better solutions when compared to the heuristic TOC-AK by Aryanezhad and Komijan (2004, p. 4221-4233). As a result, it was possible to observe that the heuristics proposed obtained a more satisfactory approach when compared to the application of TOC-AK and the identified optimum solution by Integer Linear Programming - 1.L.P. This fact evidences the importance of the heuristic proposed in the product mix definition.
\end{abstract}

Keywords

Heuristic. TOC. Product mix. 